\title{
Known and novel mutations responsible for epidermolysis bullosa simplex cases in a Chinese population
}

\author{
JIA ZHANG $^{1 *}$, YU DING $^{2 *}$, MING LI $^{1}$, ZHIRONG YAO $^{1}$ and YIN ZHUANG $^{1}$ \\ ${ }^{1}$ Department of Dermatology, Xinhua Hospital Affiliated to Shanghai Jiaotong University School of Medicine, \\ Shanghai 200092; ${ }^{2}$ Dermatology Department, Mianyang Central Hospital, Mianyang, Sichuan 621000, P.R. China
}

Received February 14, 2019; Accepted October 10, 2019

DOI: $10.3892 /$ etm.2019.8130

\begin{abstract}
Epidermolysis bullosa simplex, generalized severe (EBS-gen sev) is one of the major forms of EBS, caused by mutations of the keratin 5 (KRT5) or keratin 14 (KRT14). However, it is rarely reported in the Chinese population. The current study was performed on three unrelated Chinese families with five patients clinically suspicious for distinct stages of EBS. Mutation screening was performed by direct sequencing of the entire coding regions of KRT5 and KRT14 genes. A diagnosis of EBS-gen sev for patients in these three families was confirmed by revealing missense mutations c.373C $>$ T (p.Arg125Cys), c.374G $>$ T (p.Arg125Leu), and a novel frameshift mutation c.1231delG (p.Glu411Argfs*31) in KRT14. Considering two previously reported cases and the results of the current report, amino acid residue 125 is likely the most frequent hotspot of EBS-gen sev in the Chinese population. The current study further indicated that the symptoms of EBS-gen sev patients decline with age.
\end{abstract}

\section{Introduction}

Epidermolysis bullosa simplex (EBS) belongs to a group of inherited disorders characterized by the high occurrence of bullous lesions after a certain degree of friction or trauma. It is most commonly caused by mutations in keratin 5 (KRT5) or keratin 14 (KRT14) $(1,2)$. The protein products of these genes, keratin 5 and keratin 14, are paired intermediate filaments expressed in basal keratinocytes that contribute to mechanical stability of keratin filament networks $(1,2)$. EBS is comprised of

Correspondence to: Professor Zhirong Yao or Dr Yin Zhuang, Department of Dermatology, Xinhua Hospital Affiliated to Shanghai Jiaotong University School of Medicine, 1665 Kongjiang Road, Shanghai 200092, P.R. China

E-mail: yaozhirong@xinhuamed.com.cn

E-mail: zhuangyin@xinhuamed.com.cn

*Contributed equally

Key words: epidermolysis bullosa simplex, mutation analysis, KRT14, Chinese, prognosis three major forms: i) Localized and mild subtype, called EBS, localized (EBS-loc; OMIM 131800) or EBS Weber-Cockayne; ii) generalized and severest subtype, EBS Dowling-Meara (EBS-DM; OMIM 131760); and iii) generalized but relatively milder subtype known as EBS, generalized intermediate (EBS-gen intermed; OMIM 131900) or EBS-Koebner (3). EBS, generalized severe, formerly known as EBS Dowling-Meara (EBS-gen sev; OMIM 131760), is the most severe form of EBS. Its cardinal features include large, generalized blisters, mucous membrane involvement, and dystrophic nails. Bullous lesions are usually most severe in the neonatal and infancy stages, diminishing in severity with age, especially during later childhood and adulthood (3). The ultrastructural pathogenesis of EBS-DM includes clumping or collapsing of keratin filaments in the basal epidermal cells, leading to basal cell cytolysis and sequent intraepidermal blister formation $(4,5)$. In most cases, the clinical severity is related to the location of the mutations. Most of the mutations responsible for this subtype are located in the highly conserved $\alpha$-helical end segments of helix $1 \mathrm{~A}$ and 2B (including amino acid residue 125) of KRT5 and $K R T 14$, which are critical for proper intermediate filament structure (6). The current study reported the distinct clinical features of three Chinese probands suspected of having EBS and diagnosed with the EBS-gen sev subtype by molecular analysis.

\section{Patients and methods}

Patients. Three unrelated Chinese families with three probands clinically suspected of having EBS subtypes were enrolled in the current study at the outpatient department of Xinhua Hospital Affiliated to Shanghai Jiaotong University School of Medicine from October 2016 to October 2018, including two young males (age, 4 and 10 months), and a 19-year-old female.

$P C R$ and Sanger sequencing. Primers flanking all coding exons and intron-exon boundaries of KRT5 and KRT14 were designed using Primer Premier 5.0 software (Premier Biosoft International). Sequences of primers used in the current study are presented in Table SI. Sanger sequencing was performed to identify the mutations in all patients and to verify the sequences of unaffected family members. Peripheral blood samples of all index patients were collected in EDTA anticoagulant tubes (Insepack ${ }^{\mathrm{TM}}$; Sekisui Medical Co., Ltd.) and 
frozen at $-20^{\circ} \mathrm{C}$. TIANamp Blood DNA kit (Tiangen Biotech Co., Ltd.) was used to extract genomic DNA from 600- $\mu 1$ blood samples according to the manufacturer's instructions. Genomic DNA samples were amplified by PCR using Takara Ex Taq DNA polymerase (Takara Bio, Inc.). The following thermocycling conditions were used: Initial denaturation at $94^{\circ} \mathrm{C}$ for $5 \mathrm{~min} ; 31$ cycles of denaturation at $94^{\circ} \mathrm{C}$ for $30 \mathrm{sec}$, annealing (temperature for each primer is listed in Table SI) for $30 \mathrm{sec}$, extension at $72^{\circ} \mathrm{C}$ for $1 \mathrm{~min}$, and a final extension at $72^{\circ} \mathrm{C}$ for $1 \mathrm{~min}$; and $4^{\circ} \mathrm{C}$ for $5 \mathrm{~min}$. PCR products were purified with AxyPrep DNA Gel Extraction kit (Corning, Inc.) according to the manufacturer's instructions. The Sequencing Reaction system was based on BigDye ${ }^{\circledR}$ Terminator v3.1 Cycle Sequencing kit (Thermo Fisher Scientific, Inc.) according to the manufacturer's instructions. Purified PCR products were directly sequenced using an ABI PRISM $^{\circledR} 3730$ automated sequencer (Applied Biosystems; Thermo Fisher Scientific, Inc.).

\section{Results}

The proband in family 1 was a 10 -month-old male. His mother first came to the Department of Dermatology, Xinhua Hospital in April 2014 complaining of generalized skin blisters on the baby's hands and feet since birth (Fig. 1A and B), especially after friction or trauma. Seven months later, the skin lesions over his extremities were slightly improved (Fig. 1C). His 26-year-old father with a similar history of blistering was also present at this visit. Dermatological investigation showed no scarring, but only mild, post-inflammatory pigmentation on skin regions previously covered in blisters (Fig. 1D), although he presented with widespread blisters as an early infant.

The second proband, a 19-year-old female, presented with diffuse pigmentation, scar formation after rupture of the blisters, and scattered new blistering, resembling dermatitis herpetiformis (Fig. 1E and F). With age, the blistering diminished.

The last proband was a 4-month-old infant, with clinical manifestations similar to those of proband 1 (Fig. $1 \mathrm{G}$ and $\mathrm{H}$ ). His 25-year-old mother had a similar clinical history since birth, but currently showed only scattered blisters and mild, post-inflammatory pigmentation (Fig. 1I).

Pedigree charts of these three families are shown in Fig. 2. Mutation screening of KRT5 was negative, whereas heterozygous missense mutations c.374G $>$ T (Arg125Leu), c.373C $>$ T (Arg125Cys) and c.1231delG (p.Glu411Argfs*31) in $K R T 14$ were identified in proband 1 , proband 2 and proband 3 , respectively, and were absent in unaffected family members (Fig. 3). Mutation delineation was based on comparisons with the reported cDNA reference sequence (GenBank accession number, NM_000526.4 for KRT14). Sequencing results were analyzed using Geneious, version 5.6.7 (Biomatters, Ltd.; http://www.geneious.com/).

\section{Discussion}

The underlying pathological mechanism of EBS-gen sev is intraepidermal blister formation via basal cell cytolysis (or, rarely, acantholysis). Other subtypes can be distinguished by clumping or significant collapse of keratin filaments in the basal epidermal cells, which can be observed with immunofluorescence mapping (IFM) or electron microscopy (EM) (7). However, these primary methods may lead to discordance with the actual diagnosis (7). In addition, skin biopsy in infants is usually regarded as a somewhat unacceptable trauma for parents, and complementary genetic testing is required.

EBS-gen sev is inherited in an autosomal dominant pattern. Except for those rare cases caused by truncated mutations, including nonsense/in-frame deletion/frameshift/splicing mutations, most EBS-gen sev cases are attributed to missense mutations that exert a dominant negative effect on functional protein structure by altering inter-chain interactions (8). There is a close correlation between the mutational locus and the severity of EBS. Compared with other two major forms, EBS-loc and EBS-gen, most EBS-gen sev cases were associated with mutations in the highly conserved end segments of KRT5 or KRT14 rod domain (6), which highlights the importance of molecular diagnosis.

The site Arg125 (CGC) of KRT14 contains a CpG dinucleotide, making it a hotspot for EBS-causing mutations due to the disposition of a spontaneous mutant. Mutations in Arg125 (including Arg125His, Arg125Cys, Arg125Ser, Arg125Gly and Arg125Leu) have been shown to be responsible for at least $40 \%$ of EBS-gen sev cases. In particular, Arg125His and Arg125Cys accounted for the majority of the mutations in Arg125 (1,6,9-14). Arg125 is located in a highly conserved region and strongly perturbs keratin network formation and keratin filament assembly (15). Despite the fact that other identical sites of mutations, even in one pedigree, may lead to distinct subtypes $(1,16)$, Arg125 mutations are confined mainly to the most severe subtype EBS-gen sev with similar clinical courses. To the best our knowledge, only three pathogenic mutations of EBS-gen sev in the Chinese population (Arg165Ser in KRT5, and Arg125His and Arg125Cys in KRT14) have been reported so far. Only Arg125Leu has been reported in Korean and Polish populations. Phenotypes of patients with Arg125Leu and Arg125Cys in the current study were in accordance with the previously reported phenotypes (1,6,9-14).

Novel mutation c.1231delG (p.Glu411Argfs*31) located near the highly conserved $\alpha$-helical end segments of helix 2B could cause a stop codon in this highly conserved region, which may result in a more severe phenotype, such as EBS-gen sev. EBS harbors risk of death at an early age (17). Currently, there is no well-established cure, other than general care to prevent trauma, infection control, and good nutrition (18).

Gene therapy research of EBS is rare, although gene therapy and bone marrow transplantation for the relatively severe dystrophic or junctional epidermolysis bullosa, having the possibility of cure, have been well investigated $(19,20)$. These studies highlight that a corrective gene therapy could be an ideal therapy for EBS, however more studies are required before it can be developed and used in daily clinical practice. Prenatal or preimplantation genetic diagnosis is another sensible option for families at high risk of EBS.

The present study provided valuable information and assistance in genetic counseling such that the symptoms of EBS-gen sev patients declined with age. In clinical dermatology, a diagnosis of EBS-gen sev should be considered for patients with widespread, herpetiform, clustered blistering, especially in the neonatal period, diminishing in late childhood. It is 

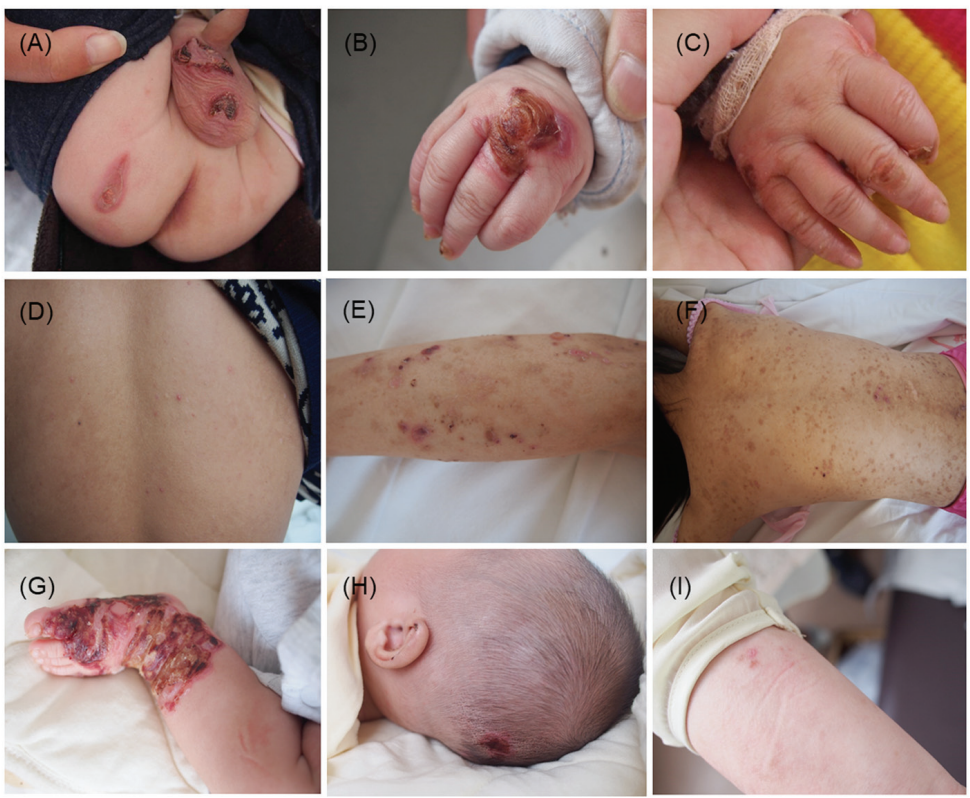

Figure 1. Clinical appearance of EBS patients. (A) The 3-month-old proband in family 1 at the first visit. (B) Proband 1 at their first visit. (C) Seven months later, bullous lesions over the extremities of proband 1 were slightly improved. (D) Twenty-six-year-old father of proband 1 showed no scarring, but only mild, post-inflammatory pigmentation on the skin regions previously covered with blisters. (E) Proband 2: A 19-year-old female presented. (F) A 19-year-old female presented with diffuse pigmentation. Scar formation occurred after rupture of blisters, accompanied with scattered new blistering. (G) Proband 3: A 4-month-old infant. (H) A 4-month-old infant with clinical manifestations similar to proband 1. (I) Twenty-five-year-old mother of proband 3 showed scattered blisters and mild post-inflammatory pigmentation where previously blistered.
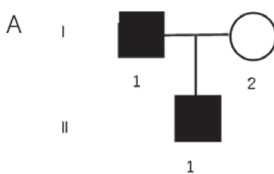

B

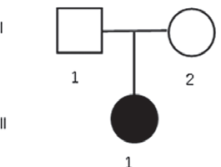

C

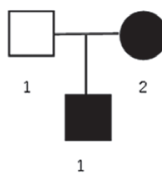

Figure 2. Pedigree charts of the three families. (A) EBS patients in family 1: A 10-month-old male and his 26-year-old father. (B) EBS patients in family 2: A 19-year-old female. (C) EBS patients in family 3: A 4-month-old infant and his 25-year-old mother. Sqaure, male; circle, female; shaded, affected; white, normal.

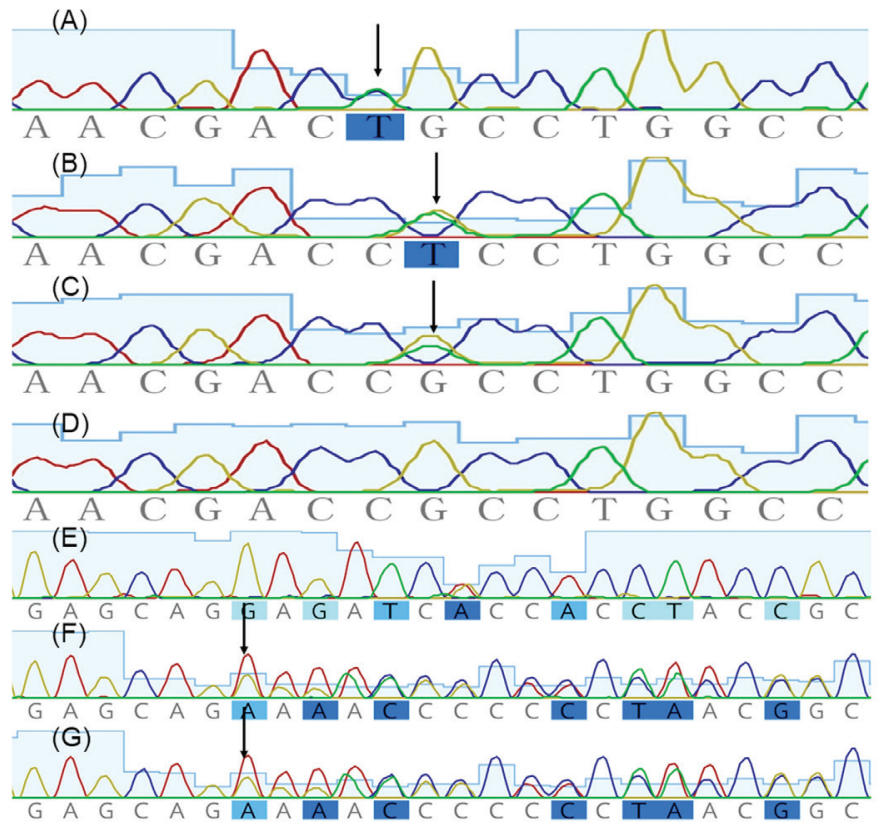

Figure 3. Sequencing results of KRT14 by Geneious, version 5.6.7 (Biomatters, Ltd.; http://www.geneious.com/). (A) A Heterozygous missense mutation, c. 373C $>\mathrm{T}$ (Arg125Cys), was revealed in the 19-year-old female in family 2. The c.374G $>\mathrm{T}$ (Arg125Leu) mutation was revealed in (B) a 10-month-old boy and (C) his father in family 1. (D) Unaffected mother in family 1. (E) Mutation c.1231delG (p.Glu411Argfs*31) in KRT14 was (E) absent in his unaffected father but appeared in $(\mathrm{F})$ the proband and $(\mathrm{G})$ his mother in family 3. Mutation delineation was based on comparing with the reported cDNA reference sequence (GenBank accession number, NM_000526.4 for KRT14). Arrows indicate the site of mutation. KRT14, keratin 14. 
generally challenging to distinguish EBS subtypes clinically in newborns. Related effective methods such as IFM and EM on freshly induced blisters combined with molecular genetic testing can be conducted to make a clear diagnosis.

\section{Acknowledgements}

Not applicable.

\section{Funding}

The present study was supported by grants from the Shanghai Sailing Program (grant no. 17YF1411900) and from National Nature Science Foundation of China (grant no. 81903197).

\section{Availability of data and materials}

The datasets used and/or analyzed during the current study are available from the corresponding author on reasonable request.

\section{Authors' contributions}

YZ and ZY conceived and designed the present study. ML collected clinical data. JZ and YD assessed the results and wrote the paper.

\section{Ethics approval and consent to participate}

The current study was approved by the Institutional Review Board of Xinhua Hospital, Shanghai JiaoTong University School of Medicine and was conducted in accordance with the principles of the Declaration of Helsinki. Ethical approval was obtained from the Ethics Committee of the Xinhua Hospital Affiliated to Shanghai Jiao Tong University School of Medicine. All participants or their legal guardians gave their written informed consent for participation.

\section{Patient consent for publication}

Patients or patients' guardians provided consent for the publication of images in the present study.

\section{Competing interests}

The authors declare that they have no competing interests.

\section{References}

1. Bolling MC, Lemmink HH, Jansen GH and Jonkman MF: Mutations in KRT5 and KRT14 cause epidermolysis bullosa simplex in 75\% of the patients. Br J Dermatol 164: 637-644, 2011

2. Fuchs E: The cytoskeleton and disease: Genetic disorders of intermediate filaments. Annu Rev Genet 30: 197-231, 1996.

3. Fine JD, Bruckner-Tuderman L, Eady RA, Bauer EA, Bauer JW, Has C, Heagerty A, Hintner H, Hovnanian A, Jonkman MF, et al: Inherited epidermolysis bullosa: Updated recommendations on diagnosis and classification. J Am Acad Dermatol 70: 1103-1126, 2014.

4. Coulombe PA, Hutton ME, Vassar R and Fuchs E: A function for keratins and a common thread among different types of epidermolysis bullosa simplex diseases. J Cell Biol 115: 1661-1674, 1991.
5. Coulombe PA, Hutton ME, Letal A, Hebert A, Paller AS and Fuchs E: Point mutations in human keratin 14 genes of epidermolysis bullosa simplex patients: Genetic and functional analyses. Cell 66: 1301-1311, 1991.

6. Yasukawa K, Sawamura D, Goto M, Nakamura H, Jung SY, Kim SC and Shimizu H: Epidermolysis bullosa simplex in Japanese and Korean patients: Genetic studies in 19 cases. Br J Dermatol 155: 313-317, 2006.

7. Hiremagalore R, Kubba A, Bansel $S$ and Jerajani $H$ : Immunofluorescence mapping in inherited epidermolysis bullosa: A study of 86 cases from India. Br J Dermatol 172: 384-391, 2015.

8. Banerjee S, Wu Q, Yu P, Qi M and Li C: In silico analysis of all point mutations on the $2 \mathrm{~B}$ domain of $\mathrm{K} 5 / \mathrm{K} 14$ causing epidermolysis bullosa simplex: A genotype-phenotype correlation. Mol Biosyst 10: 2567-2577, 2014.

9. Arin MJ, Grimberg G, Schumann H, De Almeida H Jr, Chang YR, Tadini G, Kohlhase J, Krieg T, Bruckner-Tuderman L and Has C: Identification of novel and known KRT5 and KRT14 mutations in 53 patients with epidermolysis bullosa simplex: Correlation between genotype and phenotype. Br J Dermatol 162: 1365-1369, 2010.

10. Garcia M,Santiago JL, Terron A,Hernández-Martín A, Vicente A, Fortuny C, De Lucas R, López JC, Cuadrado-Corrales N, Holguín A, et al: Two novel recessive mutations in KRT14 identified in a cohort of 21 Spanish families with epidermolysis bullosa simplex. Br J Dermatol 165: 683-692, 2011.

11. Kang TW, Lee JS, Kim SE, Oh SW and Kim SC: Novel and recurrent mutations in Keratin 5 and 14 in Korean patients with Epidermolysis bullosa simplex. J Dermatol Sci 57: 90-94, 2010.

12. Minakawa S, Nakano H, Nakajima K, Matsuzaki Y, Takiyoshi N, Akasaka E, Rokunohe D and Sawamura D: Mutational analysis on 16 Japanese population cases with epidermolysis bullosa simplex. J Dermatol Sci 72: 330-332, 2013.

13. Müller FB, Küster W, Wodecki K, Almeida H Jr, Bruckner-Tuderman L, Krieg T, Korge BP and Arin MJ: Novel and recurrent mutations in keratin KRT5 and KRT14 genes in epidermolysis bullosa simplex: Implications for disease phenotype and keratin filament assembly. Hum Mutat 27: 719-720, 2006.

14. Pfendner EG, Sadowski SG and Uitto J: Epidermolysis bullosa simplex: Recurrent and de novo mutations in the KRT5 and KRT14 genes, phenotype/genotype correlations, and implications for genetic counseling and prenatal diagnosis. J Invest Dermatol 125: 239-243, 2005.

15. Letai A, Coulombe PA, McCormick MB, Yu QC, Hutton E and Fuchs E: Disease severity correlates with position of keratin point mutations in patients with epidermolysis bullosa simplex. Proc Natl Acad Sci USA 90: 3197-3201, 1993.

16. Jankowski M, Wertheim-Tysarowska K, Jakubowski R, Sota J, Nowak W and Czajkowski R: Novel KRT14 mutation causing epidermolysis bullosa simplex with variable phenotype. Exp Dermatol 23: 684-687, 2014.

17. Hon KL, Li JJ, Cheng BL, Luk DC, Murrell DF, Choi PC and Leung AK: Age and etiology of childhood epidermolysis bullosa mortality. J Dermatolog Treat 26: 178-182, 2015.

18. Langan SM and Williams HC: A systematic review of randomized controlled trials of treatments for inherited forms of epidermolysis bullosa. Clin Exp Dermatol 34: 20-25, 2009.

19. Siprashvili Z, Nguyen NT, Bezchinsky MY, Marinkovich MP, Lane AT and Khavari PA: Long-term type VII collagen restoration to human epidermolysis bullosa skin tissue. Hum Gene Ther 21: 1299-1310, 2010.

20. Wagner JE, Ishida-Yamamoto A, McGrath JA, Hordinsky M, Keene DR, Woodley DT, Chen M, Riddle MJ, Osborn MJ, Lund $\mathrm{T}$, et al: Bone marrow transplantation for recessive dystrophic epidermolysis bullosa. N Engl J Med 363: 629-639, 2010.

This work is licensed under a Creative Commons Attribution-NonCommercial-NoDerivatives 4.0 International (CC BY-NC-ND 4.0) License. 